\title{
Tough, self-healable and tissue-adhesive hydrogel with tunable multifunctionality
}

\author{
Lu Han ${ }^{1}$, Liwei Yan ${ }^{1}$, Kefeng Wang ${ }^{2}$, Liming Fang ${ }^{3}$, Hongping Zhang ${ }^{4}$, Youhong Tang ${ }^{5}$, Yonghui Ding ${ }^{6}$, \\ Lu-Tao Weng ${ }^{7}$, Jielong $\mathrm{Xu}^{1}$, Jie Weng ${ }^{1}$, Yujie Liu ${ }^{8}$, Fuzeng Ren ${ }^{9,10}$ and Xiong Lu ${ }^{1,2,10}$
}

An ideal hydrogel for biomedical engineering should mimic the intrinsic properties of natural tissue, especially high toughness and self-healing ability, in order to withstand cyclic loading and repair skin and muscle damage. In addition, excellent cell affinity and tissue adhesiveness enable integration with the surrounding tissue after implantation. Inspired by the natural mussel adhesive mechanism, we designed a polydopamine-polyacrylamide (PDA-PAM) single network hydrogel by preventing the overoxidation of dopamine to maintain enough free catechol groups in the hydrogel. Therefore, the hydrogel possesses super stretchability, high toughness, stimuli-free self-healing ability, cell affinity and tissue adhesiveness. More remarkably, the current hydrogel can repeatedly be adhered on/stripped from a variety of surfaces for many cycles without loss of adhesion strength. Furthermore, the hydrogel can serve as an excellent platform to host various nano-building blocks, in which multiple functionalities are integrated to achieve versatile potential applications, such as magnetic and electrical therapies. NPG Asia Materials (2017) 9, e372; doi:10.1038/am.2017.33; published online 14 April 2017

\section{INTRODUCTION}

Hydrogels have a similar structure to biological soft tissues and can be engineered to resemble an extracellular matrix, and therefore have great potential for tissue engineering applications. ${ }^{1-4}$ Generally, hydrogels are brittle because of the high water content in the polymer networks, and it is a grand challenge to develop biomimetic hydrogels with superior mechanical properties. ${ }^{5}$ Several tough hydrogels have been developed, such as interpenetrating network, nanocomposite and double network hydrogels, that generally consist of interpenetrated brittle and ductile networks that can effectively dissipate energy. ${ }^{6-9}$ However, most tough hydrogels lack the ability of natural tissue to self-heal after damage because the covalent sacrificial bonds in the hydrogels cannot re-form easily when the hydrogels rupture after experiencing large deformation or cyclic loading. ${ }^{10}$ To produce highly recoverable, tough hydrogels, researchers have recently replaced sacrificial covalent bonds in the brittle networks with easily re-formable non-covalent bonds, for example, by using ionic-bond crosslinked alginate as the sacrificial network ${ }^{11}$ or using polyampholytes carrying opposite charges to create electrostatic crosslinked networks. ${ }^{12}$ The introduction of readily re-formable non-covalent bonds in the network also establishes high self-healing ability in the hydrogels. ${ }^{13}$

Hydrogels for tissue repair must also be designed with excellent cell affinity that allows cells and tissues to attach so that they can accelerate tissue regeneration after implantation. ${ }^{1,14}$ However, most reported tough hydrogels have poor cell affinity and therefore are unfavorable for cell adhesion and growth. ${ }^{15-17}$ Moreover, tough hydrogels generally lack tissue adhesion properties and therefore cannot be fixed with the surrounding tissues during surgical operation. ${ }^{1}$ Mussel-inspired hydrogels have shed new light on the development of hydrogels with good cell affinity and tissue adhesiveness. ${ }^{18-20}$ Polydopamine (PDA), which has structural similarity to the adhesive protein of mussels, has high adhesiveness to various surfaces and excellent cell affinity. ${ }^{21,22}$ PDA has also been used to prepare selfhealable hydrogels because of its highly reactive catechol groups. ${ }^{23,24}$ Most previously reported PDA-based hydrogels were formed through metal chelation, but the toxicity of metals might be a concern for biomedical applications. ${ }^{23,25}$ In brief, an ideal hydrogel for soft tissue

\footnotetext{
${ }^{1}$ Key Lab of Advanced Technologies of Materials, Ministry of Education, School of Materials Science and Engineering, Southwest Jiaotong University, Sichuan, China; ${ }^{2}$ National Engineering Research Center for Biomaterials, Genome Research Center for Biomaterials, Sichuan University, Sichuan, China; ${ }^{3}$ Department of Polymer Science and Engineering, School of Materials Science and Engineering, South China University of Technology, Guangzhou, China; ${ }^{4}$ Engineering Research Center of Biomass Materials, Ministry of Education, School of Materials Science and Engineering, Southwest University of Science and Technology, Mianyang, China; ${ }^{5}$ Centre for NanoScale Science and Technology and School of Computer Science, Engineering and Mathematics, Flinders University, Adelaide, South Australia, Australia; ${ }^{6}$ Department of Mechanical and Aerospace Engineering, The Hong Kong University of Science and Technology, Hong Kong, China; ${ }^{7}$ Department of Chemical and Biomolecular Engineering, Materials Characterisation and Preparation Facility, The Hong Kong University of Science and Technology, Hong Kong, China; ${ }^{8}$ School of Mechanics and Engineering, Southwest Jiaotong University, Chengdu, Sichuan, China and ${ }^{9}$ Department of Materials Science and Engineering, South University of Science and Technology of China, Guangdong, China

${ }^{10}$ These authors contributed equally to this work.

Correspondence: Dr F Ren, Department of Materials Science and Engineering, South University of Science and Technology of China, 1088 Xueyuan Blvd. Shenzhen, Guangdong 518055, China.

E-mail: renfz@sustc.edu.cn

or Professor X Lu, Key Lab of Advanced Technologies of Materials, Ministry of Education, School of Materials Science and Engineering, Southwest Jiaotong University, Chengdu, Sichuan 610031, China.

E-mail: luxiong_2004@163.com

Received 7 August 2016; revised 18 January 2017; accepted 23 January 2017
} 
repair should mimic the intrinsic properties of the natural counterpart, especially its high toughness and self-healing ability, in order to withstand cyclic loading and repair skin and muscle damage. In addition, excellent cell affinity and tissue adhesiveness enable integration with surrounding tissue after implantation. However, it is challenging to integrate these characteristics into one hydrogel.

Here, we designed a mussel-inspired, highly tough polydopaminepolyacrylamide (PDA-PAM) hydrogel with superior self-healing ability, excellent cell affinity and tissue adhesiveness. The key innovation of our material design is the prevention of overoxidation of PDA during hydrogel synthesis in order to maintain enough free catechol groups in the hydrogel that was inspired by the natural mussel adhesive mechanism. In nature, mussels maintain a high concentration of catechol groups for high adhesiveness by protecting catechol groups via secreting reductive cysteine-rich proteins. Having enough free catechol groups in the PDA-PAM hydrogel was the critical factor to endow unique properties, such as super stretchability, high toughness and repeatable adhesion ability, to the hydrogel. The hydrogel was tough enough to be extended to a large strain of $3300 \%$ with a fracture energy as high as $2400 \mathrm{~J} \mathrm{~m}^{-2}$. The hydrogel could fully self-heal without any external stimuli after rupture. More remarkably, the catechol groups on the PDA chains endowed the hydrogel with exceptional cell affinity and tissue adhesiveness.

\section{EXPERIMENTAL PROCEDURES}

\section{Materials}

Dopamine hydrochloride, acrylamide (AM), ammonium persulfate (APS), $N, N^{\prime}$-methylene bisacrylamide (BIS) and tetramethylethylenediamine were purchased from Sigma-Aldrich (St Louis, MO, USA). Epidermal growth factor (EGF) was purchased from Shanghai Primegene Bio-Tech (Shanghai, China). Enzyme-linked immunosorption assay (ELISA) kits were purchased from R\&D (Minneapolis, MN, USA). Fetal bovine serum, Dulbecco's modified Eagle's medium, $1 \%$ penicillin-streptomycin solution and 3-(4,5-dimethylthiazol-2yl)-2,5-diphenyltetrazolium bromide (MTT) assay were purchased from HyClone (Logan, UT, USA). All other reagents and solvents were of reagent grade.

\section{Preparation of hydrogels}

The PDA-PAM hydrogels were synthesized by the following procedures. (1) Dopamine (DA) molecules went through an alkali-induced prepolymerization process to form PDA chains by dissolving DA powder in a beaker containing $\mathrm{NaOH}$ aqueous solution $(\mathrm{pH}=11)$ and then allowing the $\mathrm{DA}$ to self-polymerize for $20 \mathrm{~min}$ in air atmosphere under stirring. (2) AM, APS BIS were mixed with the solution of PDA chains in an ice bath under stirring. After 10 min of mixing, the ice bath and stirrer were removed, and AM was polymerized to form PDA-PAM hydrogels. During AM polymerization, DA was also induced by APS to further the polymerization. Hydrogels with different weight ratios of DA/AM were synthesized to investigate the effects of DA on the properties of the hydrogels, as listed in Supplementary Table S1. Note that more APS was needed when DA was present, because DA would also be induced to second polymerization and consume APS. ${ }^{26}$ In addition, PDAPAM hydrogel did not form when the weight ratio of DA/AM was higher than $0.8 \mathrm{wt} \%$ (Supplementary Figure S1), because the reductive DA molecules affected the activity of the initiator (APS) and thus retarded the polymerization of AM monomers.

Multifunctional hydrogels hosting various nano-building blocks were also synthesized by the same polymerization process as the PDA-PAM hydrogels. Before polymerization, functional nanoparticles $\left(\mathrm{Fe}_{3} \mathrm{O}_{4} \mathrm{NPs}\right.$ and carbon black NPs) were added into the DA solution to allow DA polymerization on the nanoparticle (NP) surface. Thus, the PDA-grafted NPs could be well dispersed in the hydrogels after polymerization.
TOF-SIMS analysis. The distribution of PDA in the hydrogel (DA/AM $=5 \mathrm{wt} \%)$ and possible interaction between PAM and PDA were investigated by time-of-flight secondary ion mass spectrometry (TOF-SIMS V, ION-TOF $\mathrm{GmbH}$, Münster, Germany). The samples were bombarded with $\mathrm{Bi}^{3+}$ primary ions that were accelerated at $25 \mathrm{kV}$ with an average pulsed current of $0.3 \mathrm{pA}$. The raster area was $200 \mu \mathrm{m} \times 200 \mu \mathrm{m}$, and the acquisition time for each spectrum was $40 \mathrm{~s}$, corresponding to an ion dose of $<4 \times 10^{11}$ ion $\mathrm{cm}^{-2}$. Three positive and negative spectra were recorded for each specimen at different locations. The spectra were calibrated before extraction of the ion intensity data using the SurfaceLab 6 software (Münster, Germany).

FT-IR analysis. The FT-IR spectra of pure PAM, pure PDA and the PDA-PAM hydrogel (5 wt $\%$ DA/AM) were also analyzed to search for possible interactions between PAM and PDA using an FT-IR spectrometer (Nicolet 5700, Thermo, Waltham, MA, USA), and the spectra were recorded between 4000 and $500 \mathrm{~cm}^{-1}$.

XPS analysis. The surface chemical compositions of the PDA, PAM and PDA-PAM hydrogels were measured using XPS (Kratos, Axis Ultra DLD, Manchester, UK). A monochromatic Al Ko X-ray was used as an excitation source $(\mathrm{h} \nu=1486.6 \mathrm{eV})$ running at $15 \mathrm{kV}$ and $150 \mathrm{~W}$. The neutral C1s peak $(\mathrm{C}-\mathrm{C}(\mathrm{H})$, set at $285.0 \mathrm{eV})$ was used as a reference for charge correction. Before each measurement, the sample was washed three times in deionized water and ethanol to eliminate reactant residue from the sample and dried in a vacuum chamber at $40{ }^{\circ} \mathrm{C}$ for 2 days.

SEM characterizations. The structures of the bulk PAM hydrogel and the PDA-PAM hydrogel $(\mathrm{DA} / \mathrm{AM}=0.8 \mathrm{wt} \%)$ were examined using a scanning electron microscope (SEM, JSM 6390, JEOL, Tokyo, Japan). Before examination, the hydrogels were freeze-dried. Then, the dried hydrogels were cut to expose their inner structures, and the cross-sections were observed.

\section{Mechanical tests}

(1) Tensile tests were performed on a universal testing machine (Instron 5567, Norwood, MA, USA). The loading rate was $100 \mathrm{~mm} \mathrm{~min}^{-1}$. The specimens had a width of $25 \mathrm{~mm}$ and a thickness of $3 \mathrm{~mm}$. The gauge length between the clamps was $5 \mathrm{~mm}$.

(2) Cyclic loading-unloading tests were performed by loading the hydrogels under tension to strains of 1000 and $2000 \%$ and then unloading to zero force. A time-dependent tensile-recover-tensile test was conducted to characterize the time-dependent recoverability of the hydrogel. First, the specimens were pulled to achieve an extension ratio of 6 and then unloaded. After storage for different time intervals ( $10 \mathrm{~s}, 10 \mathrm{~min}, 20 \mathrm{~min}, 30 \mathrm{~min}$ and 1 day), the specimens were pulled to 6 times its initial length again.

(3) The fracture energy was determined by the classical single edge notch test on the universal testing machine (Instron 5567). The thickness and width of the specimen were 3 and $25 \mathrm{~mm}$, respectively (Supplementary Figure S10a). During testing, a pair of specimens was pulled: one specimen was unnotched and the other was notched (Supplementary Figure S10b). The fracture energy $\left(G_{\mathfrak{c}}, \mathrm{J} \mathrm{m}^{-2}\right)$ was calculated using the method proposed by Greensmith for elastomers. ${ }^{27}$ Details are provided in the Supplementary Information.

Rheological experiments. Dynamic rheological tests of the PDA-PAM hydrogels were characterized at room temperature using a Rheometric Scientific HAAKE (MARS, Karlsruhe, Germany) strain-controlled rheometer equipped with $20 \mathrm{~mm}$ parallel plates. The storage modulus $\left(\mathrm{G}^{\prime}\right)$ and loss tangent (ratio of loss modulus $\mathrm{G}^{\prime \prime}$ to storage modulus $\mathrm{G}^{\prime}$ ) of the hydrogel were determined as the frequency ranging from 0.01 to $10 \mathrm{~Hz}$ at $1.0 \%$ strain amplitude. Details are provided in the Supplementary Information. All rheological measurements were performed in triplicate.

Tensile-heal-tensile test. The stimulus-free self-healing behavior was visually demonstrated by a tear-heal test. The self-healing ability of the hydrogel was then quantitatively evaluated by a tensile-heal-tensile test. The hydrogel was stretched to break on the universal testing machine (Instron 5567), and the crosshead was moved back to bring the two broken pieces into contact, the two hydrogel pieces self-healed after $2 \mathrm{~h}$ and 
the healed specimen was stretched to break again. The self-healing ability was also confirmed by dynamic rheological tests using the two-step procedure. $^{18}$ Details are provided in the Supplementary Information.
Adhesion tests. To mimic the adhesiveness of hydrogels to human skin tissues, porcine skin $(25 \mathrm{~mm} \times 25 \mathrm{~mm})$ was used for the tensile-adhesion test by the universal testing machine (Instron 5567) under ambient conditions. Adhesion-strip cyclic tests were also conducted to evaluate the repeatable a

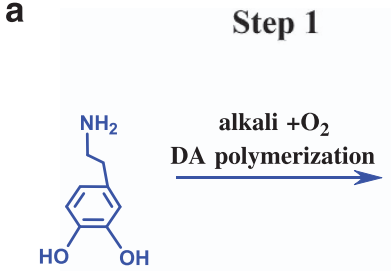

dopamine (DA)

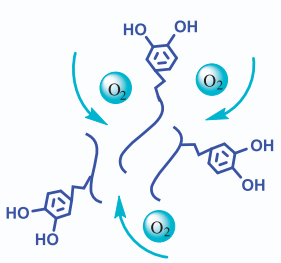

PDA chain
Step 2

preclude PDA

further oxidation

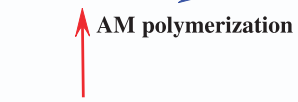

$\sim \sim \sim$

acrylamide monomer (AM)

$\mathrm{H}_{2} \mathrm{C}=\underset{\mathrm{H}}{\mathrm{C}}-\stackrel{\mathrm{C}}{\mathrm{C}}-\mathrm{NH}_{2}$

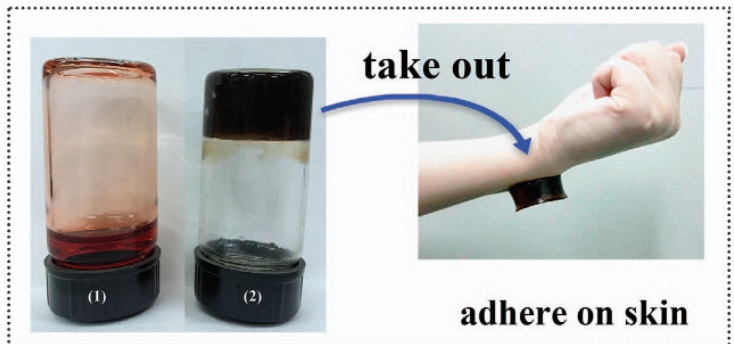

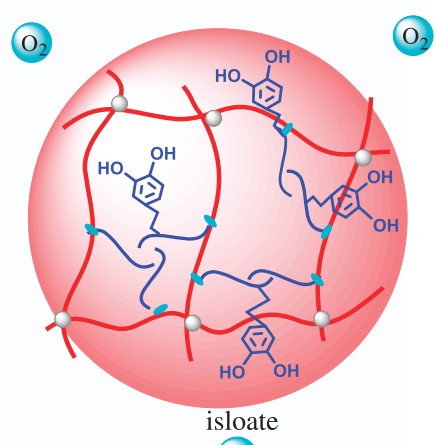

$\mathrm{O}_{2}$
$\mathrm{O}_{2}$

PDA-PAM hydrogel

b

(1)

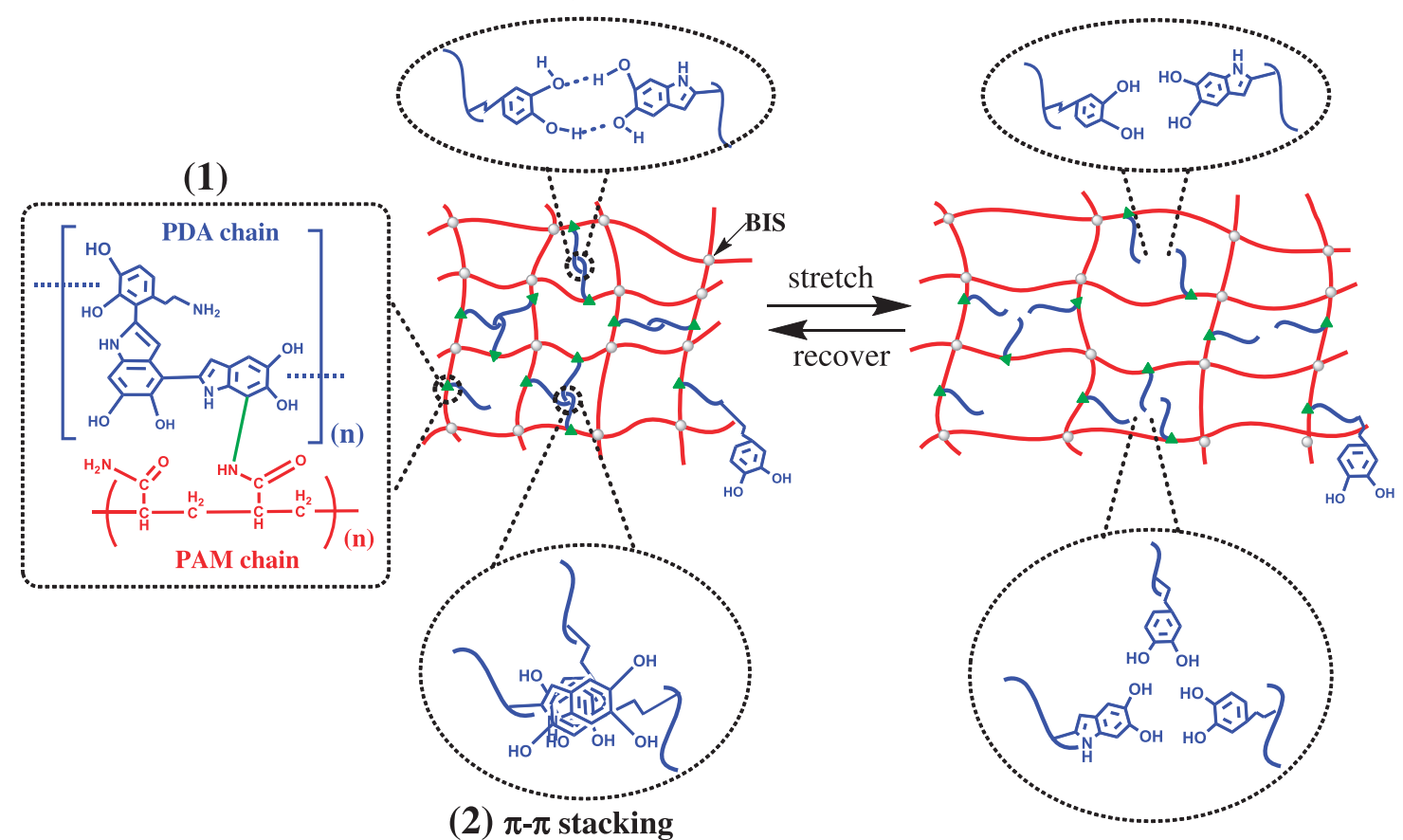

(2) hydrogen bonds

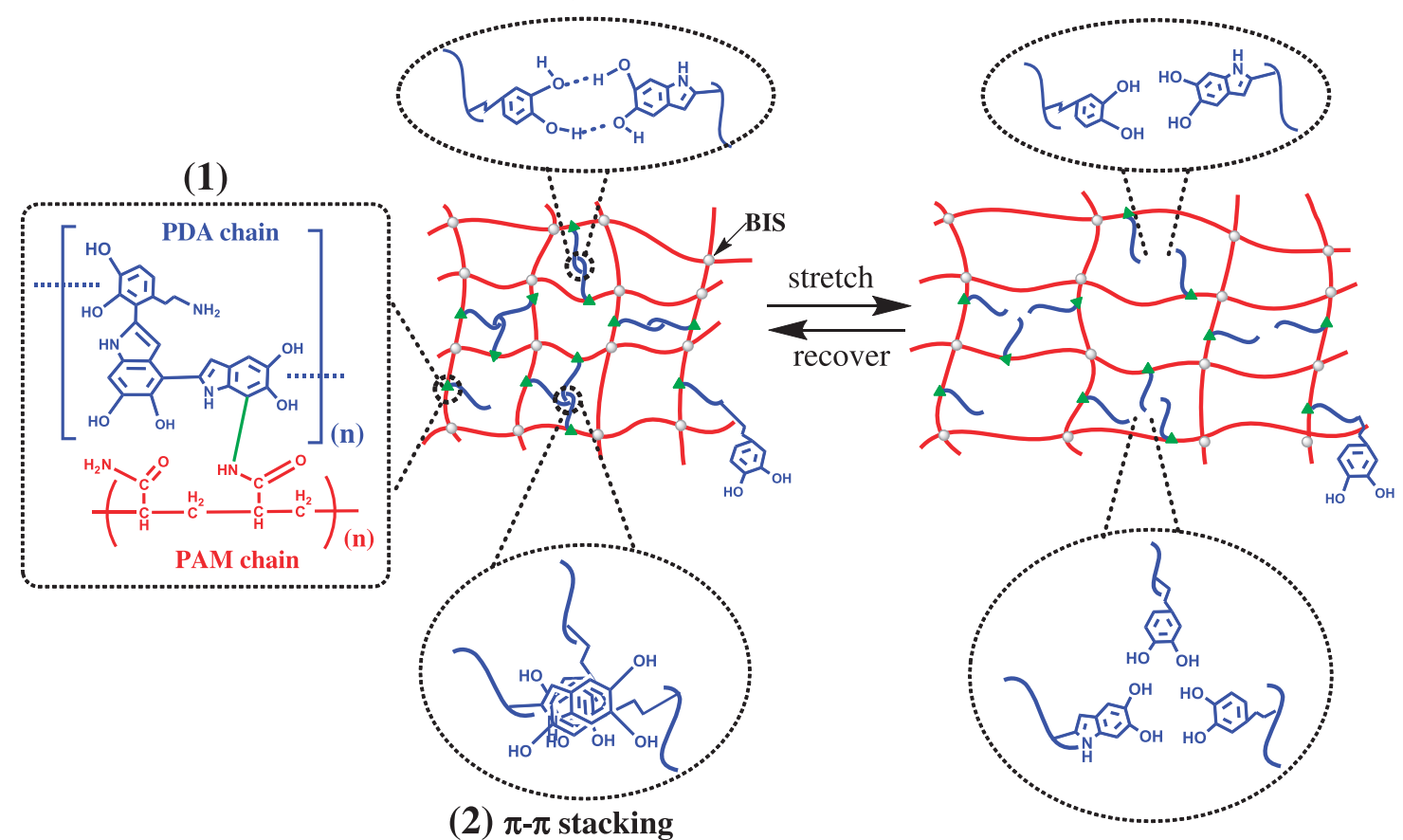

Figure 1 Synthetic process and schematic structure of the polydopamine-polyacrylamide (PDA-PAM) hydrogel. (a) The PDA-PAM hydrogel was formed by a facile two-step process. Dopamine (DA) was polymerized by oxygen to form PDA under an alkali environment. After that, acrylamide (AM) was polymerized and crosslinked to form a polymer network to obstruct external oxygen and inhibit the oxidation of catechol groups. Photo (1): without DA prepolymerization, the hydrogel could not form. Photo (2): after DA prepolymerization, the hydrogel was cured. Photo (3): the hydrogel firmly adhered on the author's arm. (b) Schematics of the molecular structure of the PDA-PAM hydrogel. The hydrogel was designed based on a single PAM network that was hybrid-crosslinked with covalent ( $N, N^{\prime}$-methylene bisacrylamide (BIS), gray circles) and reversible non-covalent bonds from PDA (black ovals). (1) The PDA chains were linked to the PAM network via interactions between free catechol groups and the amino groups of PAM (green triangles). (2) Reversible noncovalent bonds formed between the catechol groups of the PDA chains, including $\pi-\pi$ stacking and hydrogen bonds, and were diffused uniformly in the PAM network. These reversible bonds contributed to the toughness and self-healing of the single network hydrogel. 
adhesiveness of the hydrogels. The adhesion strength was calculated by the measured maximum load divided by the bonded area. Details are provided in the Supplementary Information.

Cytocompatibility evaluation. The PDA-PAM hydrogels with various DA contents $(0,0.2,0.4,0.8 \mathrm{wt} \%$ to AM) and NIH-3T3 fibroblasts (SCSP-515, Stem Cell Bank, Chinese Academy of Sciences, Shanghai, China) were used to evaluate the cell affinity of the hydrogels. The hydrogels, which had diameters of $7 \mathrm{~mm}$ and thicknesses of $2.5 \mathrm{~mm}$, were first sterilized by immersion in $75 \%$ alcohol for $24 \mathrm{~h}$. Before cell culture, the PDA-PAM hydrogels were first purified by soaking the hydrogel in deionized water three times, and the deionized water was refreshed every day. The residual amount of acrylamide monomers in the PDA-PAM hydrogel was quantified by gas chromatography. The results demonstrated that nearly no residual AM monomers existed in the PDA-PAM hydrogel after three purification cycles (Supplementary Figures S14 and S15). Then, the hydrogels were immersed in Dulbecco's modified Eagle's medium and swelled to equilibrium state for 2 days in a $\mathrm{CO}_{2}$ incubator at $37^{\circ}$ C. The biocompatibility of the hydrogel and cell proliferation were assessed by MTT assay after 3 and 7 days of culture. The morphologies of the cells on the hydrogel surfaces were observed by SEM (JSM 6390) and optical microscopy.

Full skin wounds repaired by hydrogels. Full skin wounds were created on the dorsal area of rats and treated with the purified PDA-PAM hydrogels. Five male Sprague Dawley rats weighing 180-220 g were used. After being anesthetized with pentobarbital $\left(2 \mathrm{wt} \%, 2 \mathrm{ml} \mathrm{kg}^{-1}\right)$, the dorsal area of the rat was totally depilated, and four full-thickness circular wounds $(5 \mathrm{~mm}$ in diameter) were created on the upper back of each mouse by a disposable $5 \mathrm{~mm}$ skin biopsy punch (Supplementary Figure S19). The wounds were treated with the PDA-PAM hydrogels $(\mathrm{DA} / \mathrm{AM}=0.8 \mathrm{wt} \%)$, EGF-loaded PDA-PAM hydrogels and a blank, respectively. To obtain the EGF-loaded hydrogel, the hydrogel a

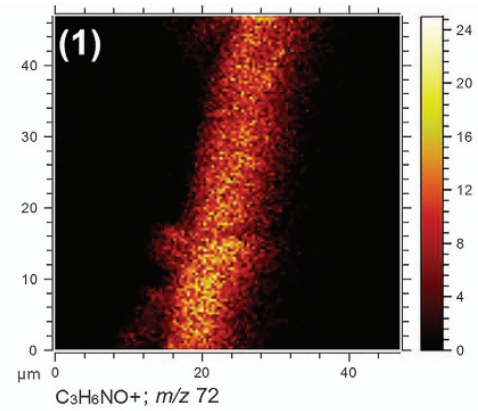

typical PAM unit<smiles>C=CC([NH-])=O</smiles>

b

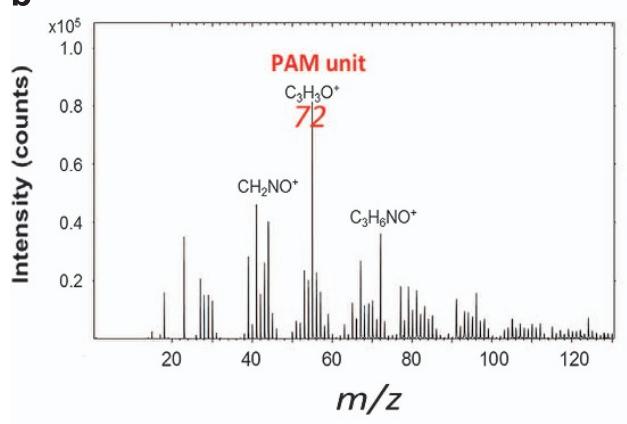

C

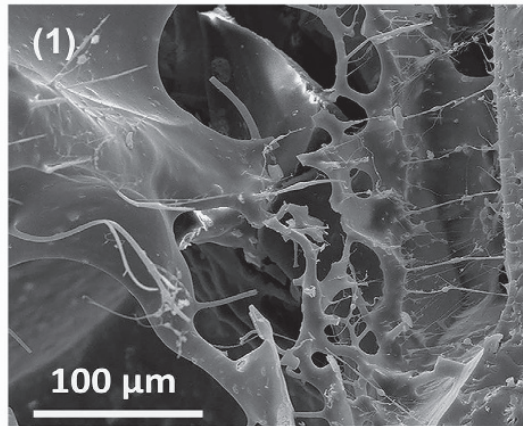

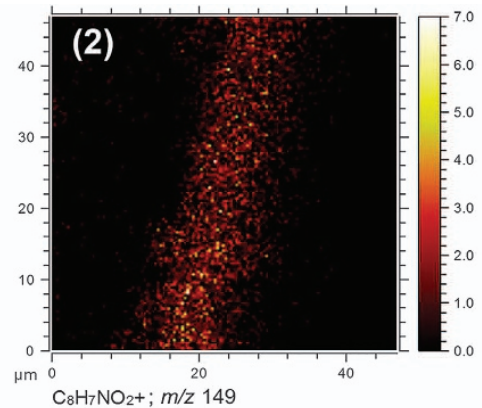

typical PDA unit
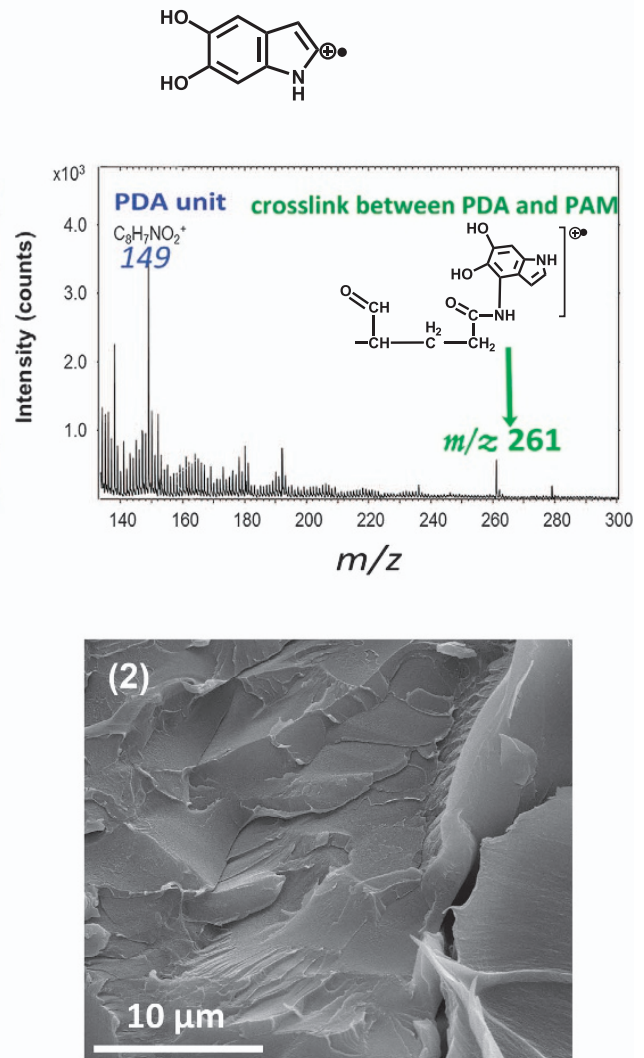

Figure 2 Characterization of the polydopamine-polyacrylamide (PDA-PAM) hydrogels. (a) Time-of-flight secondary ion mass spectrometry (TOF-SIMS) chemical maps of a cross-sectional area of the PDA-PAM hydrogel. The secondary ion images of $\mathrm{C}_{3} \mathrm{H}_{6} \mathrm{NO}^{+}\left(\mathrm{m} / z 72\right.$, left) and $\mathrm{C}_{8} \mathrm{H}_{7} \mathrm{NO}_{2}{ }^{+}(\mathrm{m} / z 149$, right) indicated the uniform distribution of PAM and PDA in the PAM-PDA hydrogel, respectively. Color intensity scales are displayed next to each image. (b) TOF-SIMS ion spectra of the PDA-PAM hydrogel. The $\mathrm{m} / \mathrm{z}$ at 72 and 149 are typical repeat units of PAM $\left(\mathrm{C}_{3} \mathrm{H}_{6} \mathrm{NO}^{+}\right)$and $\mathrm{PDA}\left(\mathrm{C}_{8} \mathrm{H}_{7} \mathrm{NO}_{2}{ }^{+}\right)$, respectively. The $\mathrm{m} / \mathrm{z}$ at 261 represents possible crosslinks between PDA and PAM. (c,1) Scanning electron microscopy (SEM) micrograph of the PDA-PAM hydrogel. $(\mathbf{c}, 2)$ SEM micrograph of the PAM hydrogel. 
(30 mg) was first sterilized in 75\% alcohol, and then EGF ( $1 \mu \mathrm{g}$ in $500 \mu \mathrm{l}$ of phosphate-buffered saline) was absorbed into the hydrogel. After 0,5 and 15 days post wounding, photographs of the wound closure were taken for each wound with a digital camera, and the percentages of wound closure were calculated. All rats were killed on day 15, and the wound site with the surrounding skin was harvested. Central wound sections were fixed on glass slides and stained with hematoxylin and eosin and Masson's trichrome to evaluate the wound-healing progress and investigate the skin tissue regeneration and collagen deposition. The experiments were performed in accordance with protocols approved by the local ethical committee and laboratory animal administration rules of China.

\section{RESULTS AND DISCUSSION}

\section{Hydrogel synthesis and characterization}

The hydrogel was synthesized by a facile two-step synthetic method (Figure 1a). The first step was an alkali-induced PDA polymerization, in which DA molecules were oxidized to form PDA chains through reaction of the catechol and quinone groups. With the polymerization of PDA, the solution underwent a color change from colorless to pale brown and finally turned to deep brown black over time, which is the typical color of PDA. ${ }^{28}$ Note that this step was critical to allow the following gel formation process because free DA monomers strongly retarded AM polymerization and thus gelation could not occur (inset photo in Figure 1a). In the second step, acrylamide monomers were added to the above PDA precursor solution and polymerized to form the PDA-PAM hydrogel in the presence of initiator and crosslinker. Importantly, this step prevented further oxidation of PDA by isolating unreacted catechol groups from the outside oxygen environment, and thus enough free catechol groups remained in the hydrogel. PDA-PAM hydrogels with different DA weight ratios were prepared as listed in Supplementary Table S1.

In the PDA-PAM hydrogel, PDA chains were linked to the PAM network through interactions between the catechol groups of the PDA chains and the amino groups of the PAM network (Figure 1b). The PDA chains intertwined to form recoverable non-covalent bonds, including $\pi-\pi$ stacking and hydrogen bonds, ${ }^{26,28,29}$ dispersed in the PAM network. Thus, the PAM network was hybrid crosslinked by covalent bonds and reversible non-covalent bonds from PDA. In addition, the interaction between PDA chains and the PAM network led to load sharing between the ductile PAM network and the non-covalent bonds in the crosslinked PDA chains. Consequently, the PAM network could significantly extend to sustain large deformations and maintain the elasticity of the hydrogel. The reversible bonds from the PDA chains linked to the PAM network could dissipate energy efficiently through breakage of the non-covalent bonds to prevent crack propagation during stretching, therefore enhancing the toughness and increasing the extension ratio as well as endowing the hydrogel with excellent self-healing ability. ${ }^{13}$

The interaction between PDA and PAM in the PDA-PAM hydrogel was confirmed by TOF-SIMS, FT-IR, proton nuclear magnetic resonance and XPS (Figure 2b and Supplementary Figures S2-S5). The chemical maps of TOF-SIMS indicated a homogenous dispersion of PDA in the PAM network (Figure 2a). The secondary ions $\mathrm{C}_{3} \mathrm{H}_{6} \mathrm{NO}^{+}$ $\left(m / z\right.$ 72) and $\mathrm{C}_{8} \mathrm{H}_{7} \mathrm{NO}_{2}{ }^{+}(\mathrm{m} / z$ 149) were chosen to represent PAM and PDA, respectively. These two ions were uniformly distributed over the whole cross-section area, suggesting the uniform distribution of PAM and PDA components in the hydrogel. A peak at $m / z 261$ was observed in the positive spectrum of the PDA-PAM hydrogel, but it did not appear in either the PAM spectrum or the PDA spectrum (Supplementary Figures S2a and b). This suggests that the peak at $m / z$ 261 originates from the reaction between PAM and PDA. It has been reported that the catechol/quinone groups on PDA could crosslink with nucleophilic amine groups via a Schiff base reaction. ${ }^{28,30,31}$ In agreement with this notion, the tentative assignment of the peak at $m / z 261$ is $\mathrm{C}_{13} \mathrm{H}_{14} \mathrm{~N}_{2} \mathrm{O}_{4}{ }^{+}$(see the inset of Figure 2b) that results from interactions between the amine groups of PAM and the catechol groups of PDA. This reaction connects the PDA chains to the PAM networks. In addition, the FT-IR spectrum of the PDA-PAM hydrogel showed a new peak at $1258 \mathrm{~cm}^{-1}$, corresponding to $\mathrm{C}-\mathrm{N}$ stretching in phenyl amines, in comparison with pure PAM and PDA (Supplementary Figure S3). The presence of this peak also indicated interactions between the $-\mathrm{NH}_{2}$ groups of PAM and the catechol groups of PDA, as shown in the black square in Figure $1 \mathrm{~b}(1)$. Proton nuclear magnetic resonance analysis was also used to investigate the interactions of DA and AM (Supplementary Figure S4). After AM was mixed with DA to form AM-DA complexes, the broad band of the catechol groups in DA (8.87 p.p.m.) split into two sharp peaks (8.85 and 8.88 p.p.m.). In addition, the active hydrogen in DA shifted from 7.99 to 7.8 p.p.m. These results demonstrated that there were interactions between the oligomers of DA and AM. XPS analysis also confirmed the presence of free catechol groups in the PDA-PAM hydrogel (Supplementary Figure S5). SEM micrographs showed that the PDA-PAM hydrogels displayed microfibril structures, whereas the pure PAM hydrogel had a smooth surface (Figure $2 \mathrm{c}$ and Supplementary Figure S6). The microfibrils in the hydrogel may be caused by the complexation of PDA and PAM chains that led to interweaved three-dimensional structures.

\section{Stretchability, toughness and fatigue resistance}

The PDA-PAM hydrogel exhibited super stretchability and high toughness. The hydrogel was stretched 30 times beyond its initial length, as shown in the typical tensile stress-strain curves (Figures 3a and b). Note that the stress-strain curves showed the nonlinear inelastic mechanical behavior of the hydrogel, and the Young's modulus (E) decreased during extension, and this is the widely encountered softening phenomenon of highly stretchable hydrogels. ${ }^{7}$ Increasing the content of DA remarkably enhanced the stretchability of the hydrogels (Figure 3c). The tensile loading-unloading tests showed pronounced hysteresis, indicating energy dissipation from bond breakage. In addition, during tensile loading-unloading tests, the residual strain was very small, even when the hydrogel was stretched to a strain of $2000 \%$ (Figure 3d). After removal of the load, the hydrogel automatically recovered its original shape (Supplementary Figure S8). However, if the second loading was applied immediately $(10 \mathrm{~s})$, the strength of the hydrogel was slightly weaker. After sufficient storage duration (1 day), when stretched again, the hydrogel showed almost full self-recovery. In contrast, the pure PAM hydrogel ruptured, rather than recovered, during the second tensile test (Supplementary Figure S9). These results indicated that the internal damage of the hydrogel showed fatigue resistance and could withstand cyclic loading after the addition of DA, although the recovery process took time.

The fracture energy of the PDA-PAM hydrogel $\left(2400 \mathrm{~J} \mathrm{~m}^{-2}\right)$ is much higher than that of the pure PAM hydrogel $\left(650 \mathrm{~J} \mathrm{~m}^{-2}\right)$ (Figure 3e) and human cartilage $\left(\sim 1000 \mathrm{~J} \mathrm{~m}^{-2}\right),{ }^{11}$ as measured by single-edge notched tests (Supplementary Figure S10). In particular, during the experiment, in contrast to the notch-sensitive pure PAM hydrogels, where the notch quickly turned into a running crack during stretching, the PDA-PAM hydrogels were insensitive to the notch and could be stretched to a large fracture strain of 26 (Supplementary Videos S1 and S2). The PDA-PAM hydrogel was also tough enough to bear stretching after being knotted (Figure 3f) and recovered its original shape even when subjected to compression strains of up to 
a
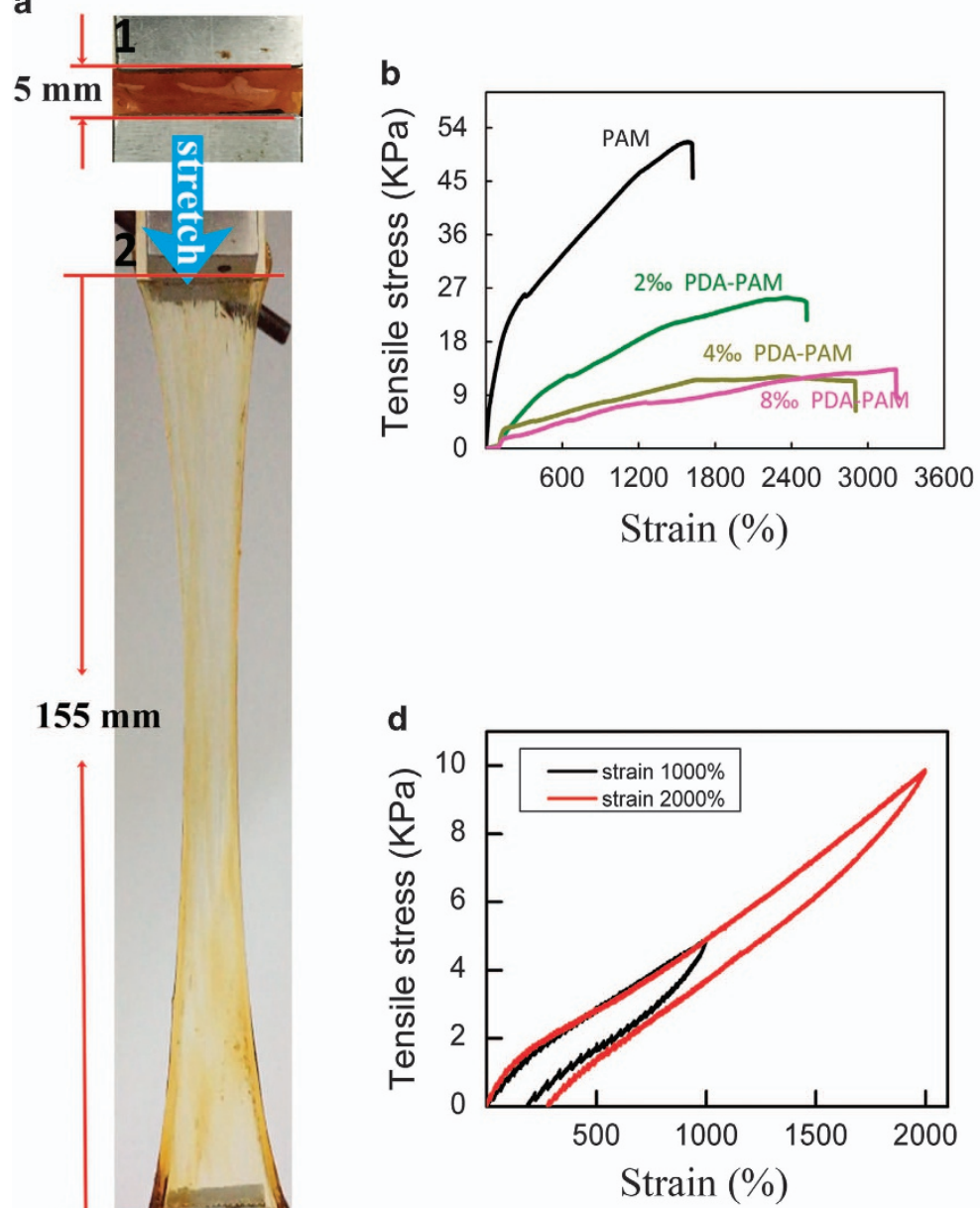

C

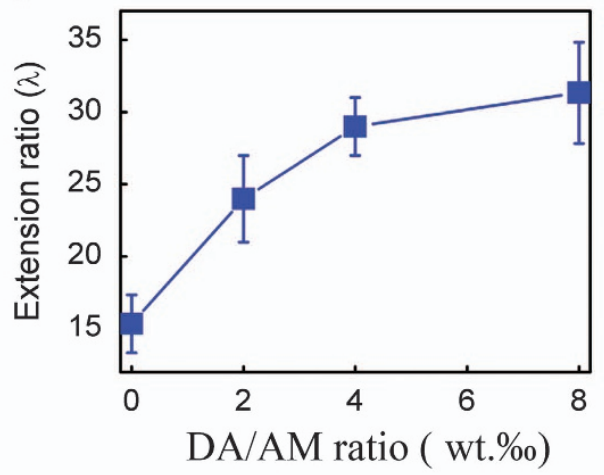

e

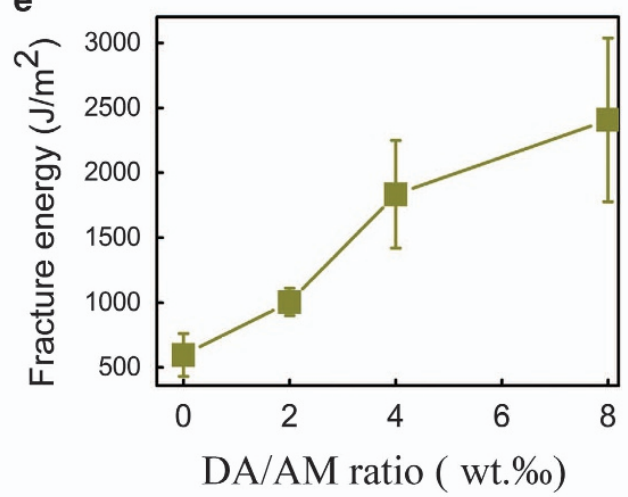

f

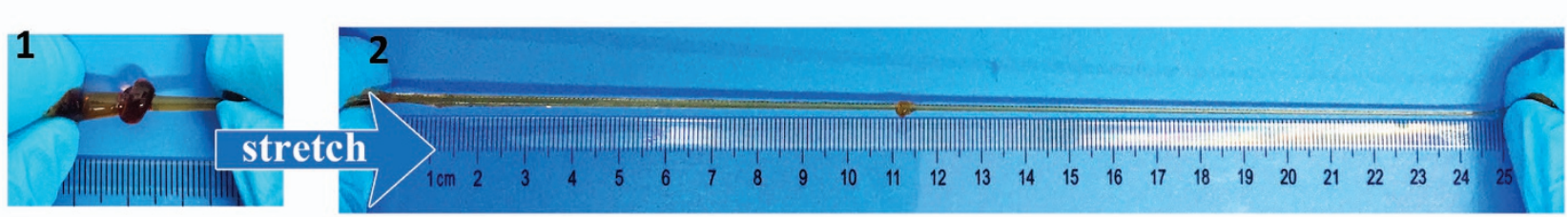

$\mathbf{g}$

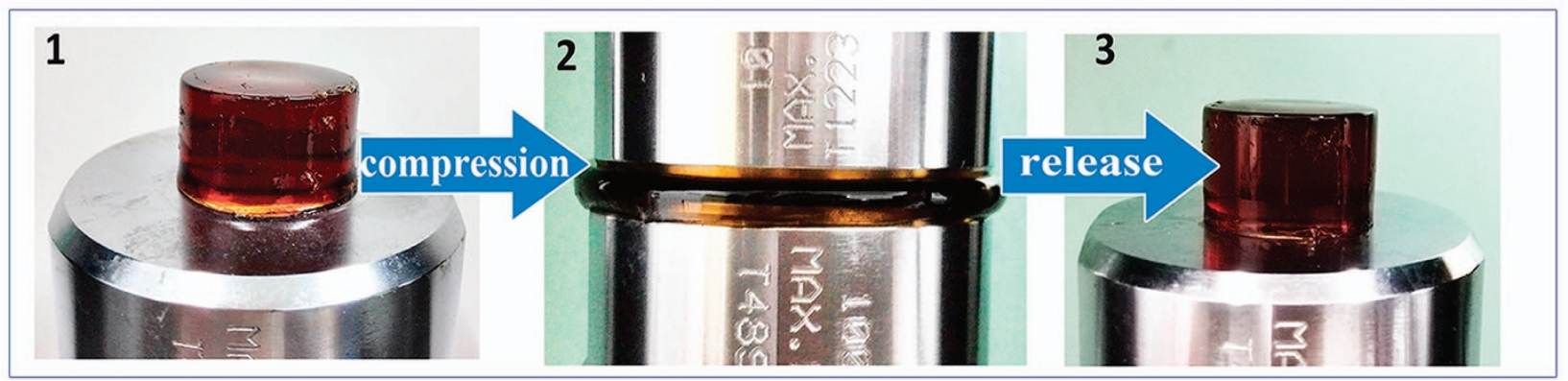

Figure 3 The effect of dopamine (DA) content on the mechanical properties of the hydrogels. (a) A typical photograph of the tensile test in which super stretchability was demonstrated. The polydopamine-polyacrylamide (PDA-PAM) hydrogel (0.8 wt\% DA/acrylamide (AM)) was stretched to 31 times its initial length. (a1) The initial shape of the hydrogel with a gauge length of $5 \mathrm{~mm}$ and (2) the stretched shape of the hydrogel at $3100 \%$ strain. (b) Typical tensile stress-strain curves of the hydrogels with different DA/AM ratios. (c) Maximum extension ratio of the hydrogels with different DA/AM ratios. (d) Two hydrogels with DA/AM ratios of $0.8 \mathrm{wt} \%$ were subjected to the loading-unloading cycle tests with large strains of 1000 and $2000 \%$. (e) The fracture energy of the hydrogels as a function of DA/AM ratio. (f) The hydrogel (DA/AM $=0.8 \mathrm{wt} \%$ ) could still bear stretching to 8 times its initial length after it was knotted. (g) The recoverability of the hydrogel (DA/AM $=0.8 \mathrm{wt} \%$ ) under compression. (1) The initial shape of the cylindrical specimen (12 $\mathrm{mm}$ in diameter, $10 \mathrm{~mm}$ in height); (2) the shape under compression of up to $85 \%$ strain; and (3) the PDA-PAM hydrogel recovered its initial shape after releasing the compression load. 
a

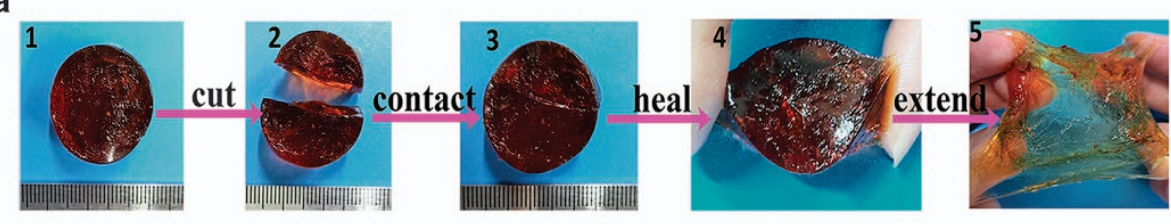

b

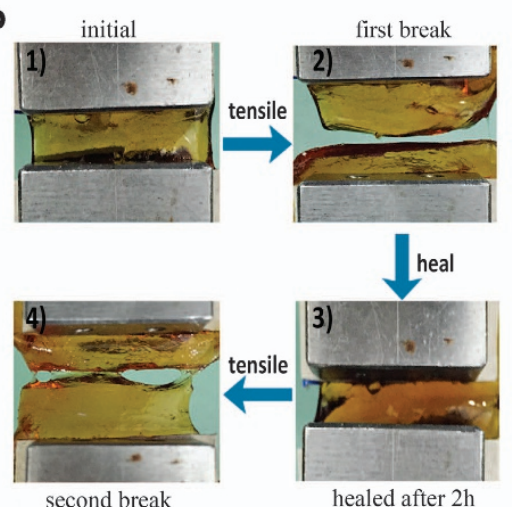

c
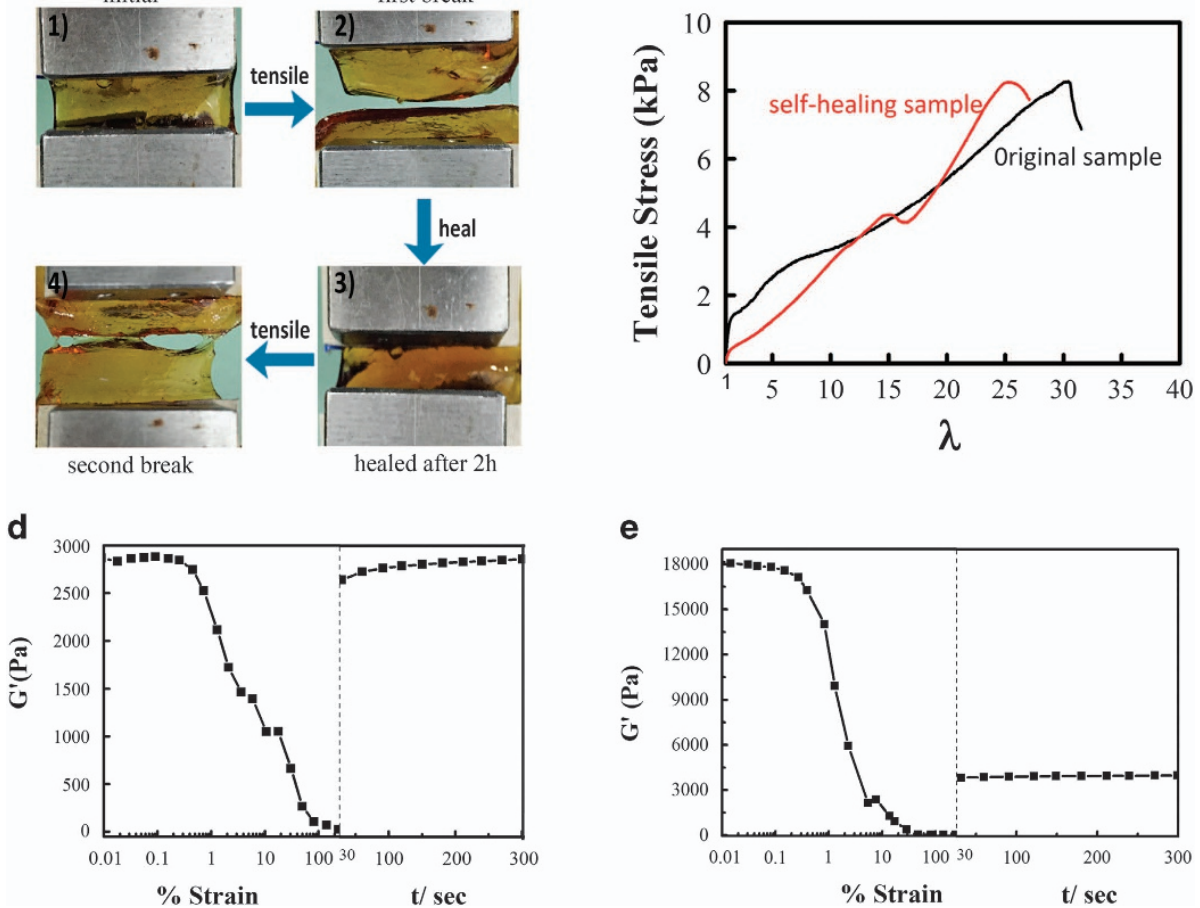

e

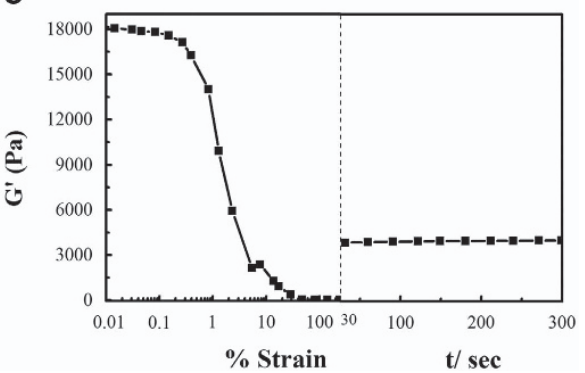

Figure 4 The self-healable polydopamine-polyacrylamide (PDA-PAM) hydrogel. (a) Tear-heal test. (1,2) The PDA-PAM hydrogel (0.8 wt\% dopamine/ acrylamide (DA/AM)) was torn into two pieces; (a3) the separated pieces were brought back into contact for self-healing; (4) the two parts rejoined after $30 \mathrm{~min}$; and (5) the fracture trace could not be identified after the hydrogel was stretched. (b) Tensile-heal-tensile test. (1) Initial shape; (2) the specimen was stretched to break, and the crosshead was moved back to bring the two broken pieces into contact; (3) the two pieces self-healed after $2 \mathrm{~h}$; and (4) the healed specimen was stretched to break again. (c) Typical tensile stress-extension ratio curves of the initial and self-healed PDA-PAM hydrogels. Dynamic rheological experiments illustrated the self-healing properties of (d) the PDA-PAM hydrogel (0.8 wt\% DA/AM) and (e) the pure PAM hydrogel.

$85 \%$ (Figure 3g). However, the addition of DA decreased the tensile strength (Supplementary Figure S7). These phenomena could be ascribed to the increase in the viscosity of the hydrogels after DA addition (Supplementary Figure S11), as evidenced by dynamic rheological tests, in which the loss tangent $\left(G^{\prime \prime} / G^{\prime}\right)$ increased with DA content. The crosslinker (BIS) content affected the mechanical properties of the hydrogels (Supplementary Tables S2 and S3).

\section{Stimuli-free self-healing properties}

The PDA-PAM hydrogel had self-healing ability in the ambient environment without any metal-chelation agents and/or other external stimuli that was ascribed to catechol-mediated interfacial hydrogen bonds and $\pi-\pi$ stacking (Figure $1 \mathrm{~b}$ ). As shown in Figure $4 \mathrm{a}$, when we tore the hydrogel into two separate pieces and then brought them into contact, they automatically rejoined after $2 \mathrm{~h}$ in the ambient environment. The fracture line could not be identified when the healed hydrogel was further stretched. To quantitatively evaluate the self-healing property, we performed a tensile-heal-tensile test (Figure $4 \mathrm{~b}$ ). The healed hydrogel could once again sustain a large strain after a healing duration of $2 \mathrm{~h}$ at room temperature. No large differences were observed between the two tensile stress-extension ratio curves, indicating that the hydrogel had fully recovered
(Figure 4c). The self-healing property was further confirmed by dynamic rheological tests (details in the Supplementary Information). A $100 \%$ strain through a strain amplitude sweep was first applied to break the hydrogels, and subsequently a low strain $(0.01 \%)$ amplitude and low frequency $(\mathrm{f}=1 \mathrm{~Hz})$ was applied to allow the hydrogel to recover. As shown in Figure $4 \mathrm{~d}$, the storage modulus G' (2869 Pa) of the recovered PDA-PAM hydrogel nearly recovered its initial value $(2707 \mathrm{~Pa})$ within $30 \mathrm{~s}$ after stopping the large strain, whereas the pure PAM hydrogel did not recover its initial $G^{\prime}$ value (Figure 4e). Note that the hydrogel could perfectly self-heal if two freshly cut surfaces were maintained in contact for $2 \mathrm{~h}$. In contrast, if two uncut surfaces were brought together, they could adhere to each other and be separated by pulling (Supplementary Figure S12c). This is because the freshly cut surfaces have many active catechol groups that lead to strong self-healing, whereas the catechol groups on uncut surfaces can lead to adhesion that is different from previous self-healing hydrogels based on host-guest interactions. ${ }^{32}$ These results thereby confirm the reversible nature of the non-covalent bonds between PDA chains that can re-form after breakage and therefore impart self-healing properties to the PDA-PAM hydrogel..$^{13}$ 
a
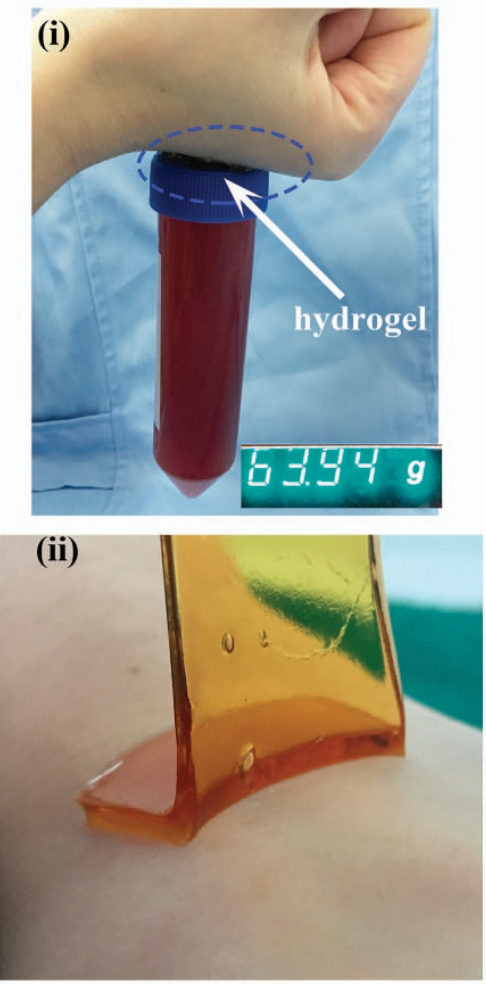

C

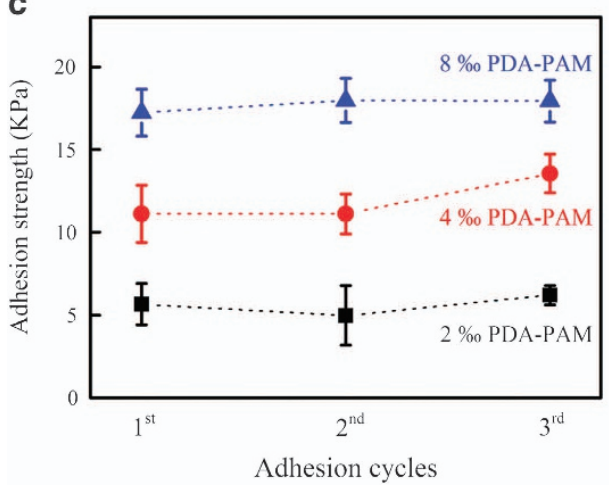

b
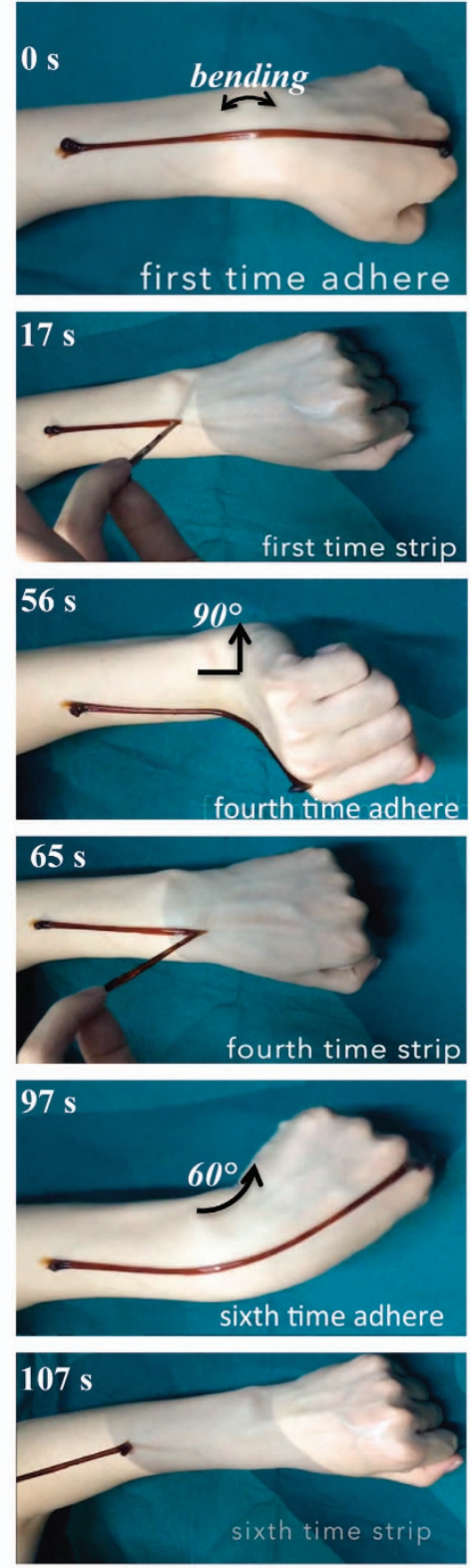

Figure 5 Tissue adhesiveness of the polydopamine-polyacrylamide (PDA-PAM) hydrogel. (a,i) The hydrogel could tightly adhere on the author's hand and hang a tube full of water with a weight of $63.94 \mathrm{~g}$. (a,ii) Photos of the peeling process of adhesive hydrogel adhered on the author's skin. (b) Photos of the repeatable adhering-peeling process of a slope-like PDA-PAM hydrogel on the author's arm, showing that the hydrogel could repeatedly be adhered on/ stripped from human skin multiple times, even with bending or twisting of the wrist, and the hydrogel could be easily peeled off without hurting the skin. (c) The adhesive strength of the PDA-PAM hydrogel to porcine skin tissue surfaces over multiple adhesion-strip cycles. The adhesive strength of the hydrogel did not attenuate even after several cycles of the adhere-strip process.

\section{Tissue adhesiveness}

The PDA-PAM hydrogel possessed exceptional tissue adhesiveness that provided universal soft tissue adhesion with no skin tissue irritation or inflammatory response. As shown in Figure 5a,i, the hydrogel tightly adhered on the author's skin and could support a tube full of water. The hydrogel could be easily peeled off without leaving any residue on the skin when no longer in use (Figure 5a,ii). The adhesiveness of the hydrogel herein allowed it to conform readily to dynamic skin surfaces. As shown in Figure 5b, the hydrogel still was firmly attached on the author's skin when it was stretched and followed the movement of the joint as the wrist was bent or twisted.
The hydrogel had repeatable and superior adhesiveness even after six cycles of adhere strip, and the skin was not hurt during the adhere-strip cycle (Figure 5b and Supplementary Video S3). The adhesive strength of the hydrogel to porcine skin increased with DA content and reached a maximum of $15.2 \mathrm{KPa}$ when the DA/AM ratio was $0.8 \mathrm{wt} \%$. Almost no loss of adhesive strength was measured during the adhesion-strip cyclic test (Figure $5 c$ ) that revealed that the adhesion was reversible, thereby benefiting the removal of the hydrogel when it was no longer needed. The free catechol groups in PDA could interact with amine or thiol groups to form cation $-\pi$ or $\pi-\pi$ interactions with various contact surfaces ${ }^{1,33}$ that were responsible 
a

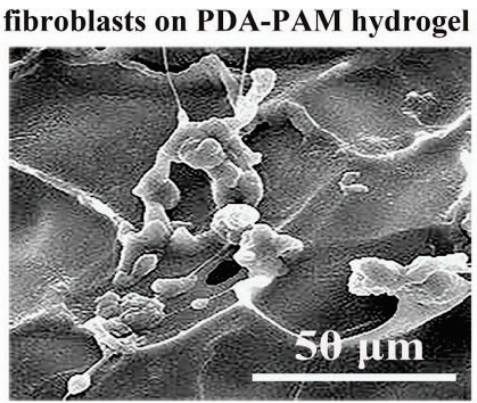

C

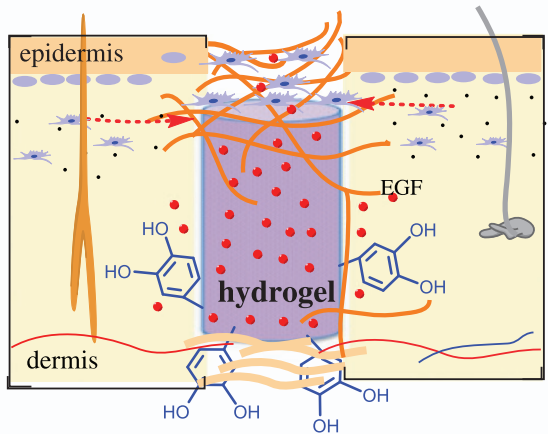

e

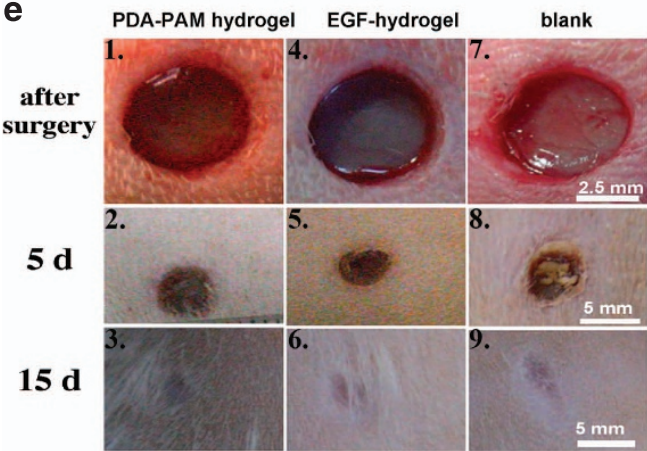

b

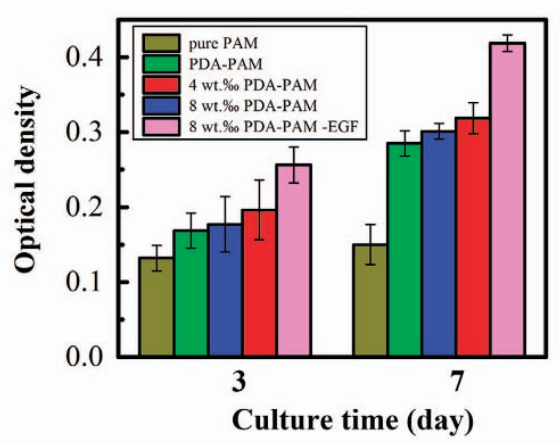

d

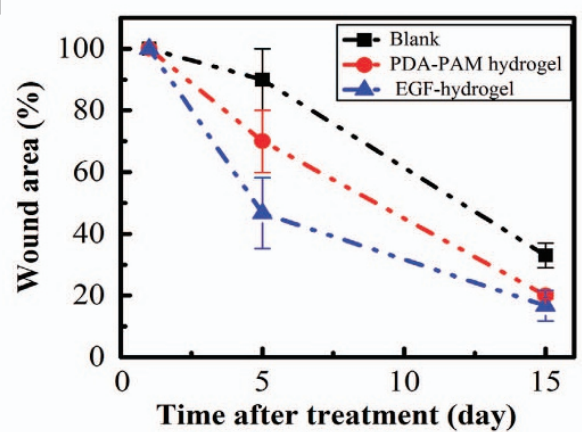

f

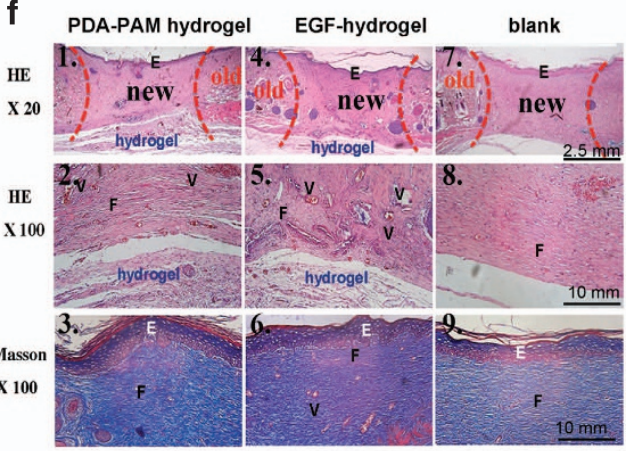

Figure 6 The cell affinity and tissue repair of polydopamine-polyacrylamide (PDA-PAM) hydrogels. (a) Scanning electron microscope (SEM) morphology of fibroblasts adhered to the PDA-PAM hydrogel (0.8 wt\% dopamine/acrylamide (DA/AM)). (b) Fibroblast density of the hydrogels by 3-(4,5-dimethylthiazol-2-yl)2,5-diphenyltetrazolium bromide (MTT) measurement. (c) Schematic of the full skin defect model to evaluate skin tissue regeneration. (d) Open wound area (\% of initial area) for each group. (e) Representative macroscopic appearance of the hydrogels implanted in the skin wound after surgery and wound closure. (f) Histological evaluation of wounds treated by hydrogels after 15 days. (1, 4 and 7) Hematoxylin and eosin (H\&E) staining shows the general arrangement of hydrogel and newly regenerated skin tissue. (2, 5 and 8) Magnified (H\&E staining) images of the bottom area of 1, 4 and 7, respectively, show that the hydrogels are well integrated with the skin tissue. (3, 6 and 9) Magnified images (Masson staining) of the top area of 1, 4 and 7, respectively. E, epidermis; F, fibroblast; EGF, epidermal growth factor; $V$, vessel; new, newly regenerated skin tissue; old, intact skin tissue surrounding the wound.

for the adhesion properties. Note that more BIS reduced the adhesion strength (Supplementary Figure S13), because the increase in crosslinking density of the hydrogel restricted the mobility of the polymer chains. ${ }^{34}$ Consequently, the viscosity of the hydrogel decreased, and the polymer chains were not easily diffused into the tissue surface to form intimate contact with the tissue surfaces.

\section{Biocompatibility and wound repair}

Both the in vitro cell culture and in vivo animal experiments demonstrated that the PDA-PAM hydrogel was biocompatible. The hydrogel had high affinity to cells because the catechol groups can form strong interactions with functional groups in the cell membrane, such as thiols and imidazoles. ${ }^{1}$ As shown in Figure 6a and Supplementary Figure S16, a large number of fibroblasts adhered to and spread well on the surfaces of the PDA-PAM hydrogels. Increasing the DA content in the hydrogels enhanced adhesion and promoted the proliferation of fibroblasts (Figure $6 \mathrm{~b}$ ).

In vivo implantation demonstrated that the PDA-PAM hydrogels could accelerate skin tissue regeneration (Figures $6 c-f$ ). Surgery details are provided in the Supplementary Figure S19. The wounds treated with PDA-PAM hydrogels were covered by complete and thick epidermis (Figures 6e,3 and f,1). The regenerated new skin tissue integrated well with the PDA-PAM hydrogels (Figure 6f,2), and they were mature, as evidenced by well-organized and aligned collagen fibers in the wound area (Figure 6f,3). After EGF was loaded in the PDA-PAM hydrogel, the wound was completely repaired with hair coverage (Figure 6e,6). The interface between the newly regenerated skin tissue and the hydrogel was diffused 
a

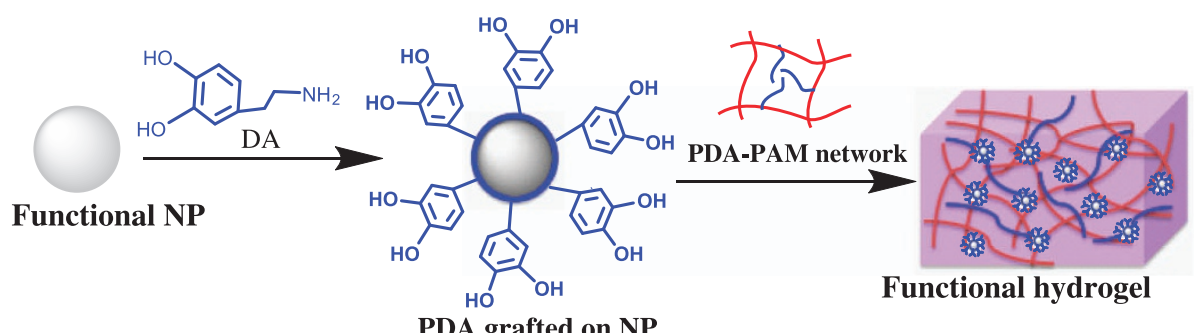

b

Conductive, stretchable, and self-healable hydrogel

(i)

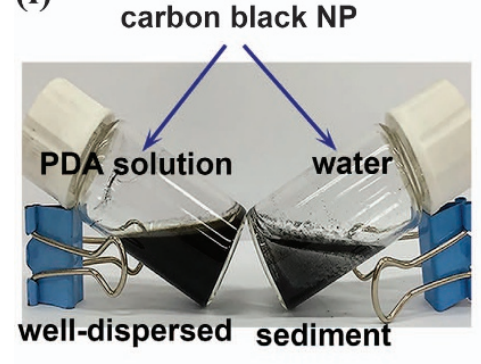

(ii)

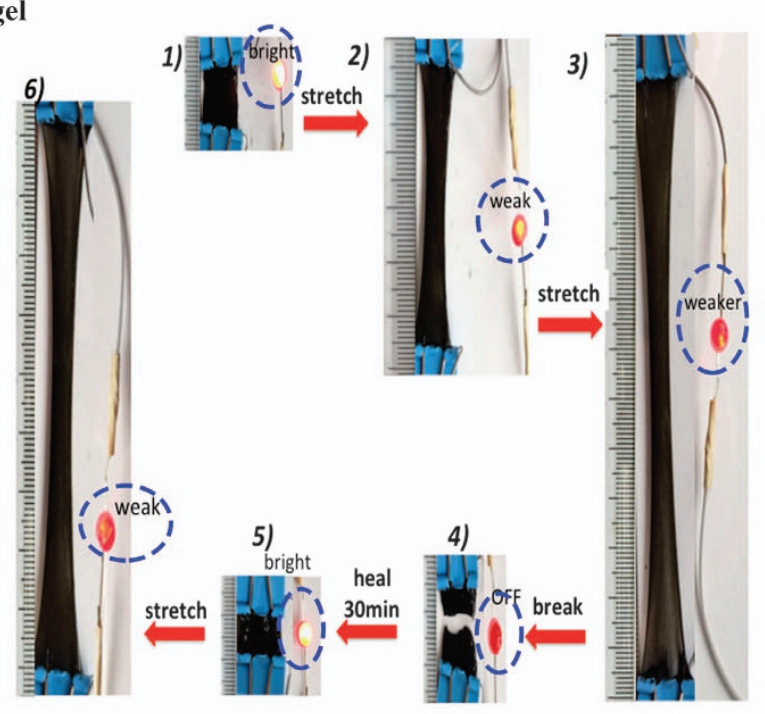

c

Magnetic, adhesive, and stretchable hydrogel

(i)

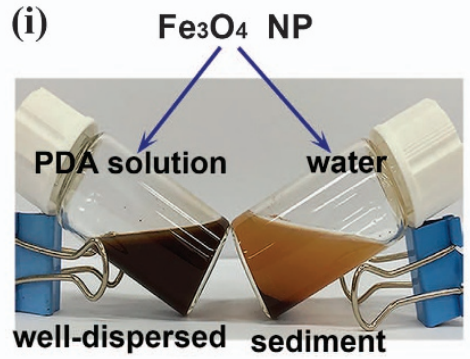

(ii)

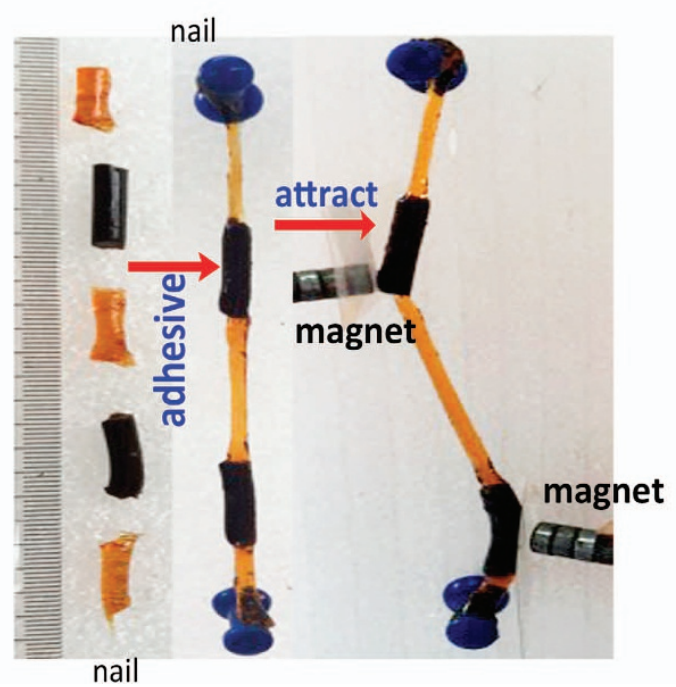

Figure 7 Magnetic and conductive hydrogels incorporated with functional nanoparticles. (a) Schematic of polydopamine (PDA)-grafted nanoparticles (NPs) to functionalize the polydopamine-polyacrylamide (PDA-PAM) hydrogel. (b) The conductive, stretchable and self-healable hydrogel with carbon black (CB) NPs. (i) PDA-grafted NPs were well dispersed in the PDA solution $\left(2 \mathrm{mg} \mathrm{ml}^{-1}\right.$ ) (left), whereas pure NPs settled at the bottom of the water (right). (ii) The hydrogel had good conductivity while still maintaining its self-healing properties and stretchability in a circuit. The brightness of the light-emitting diode (LED) light varied during the process. (c) The magnetic, adhesive and stretchable hydrogel with $\mathrm{Fe}_{3} \mathrm{O}_{4} \mathrm{NPs}$. (i) $\mathrm{Fe}_{3} \mathrm{O}_{4} \mathrm{NPs}_{\text {s }}$ in PA solution. (ii) A hydrogel rope formed by self-adhering two magnetic hydrogel blocks with $\mathrm{Fe}_{3} \mathrm{O}_{4} \mathrm{NPs}$ (brown) and three pure hydrogel blocks (yellow) that was attracted and bent by two magnets.

(Figure 6f,5), and more mature and compact collagen fibers were present (Figure 6f,6).

These in vivo wound-healing results indicated that the PDA-PAM hydrogel facilitated a quicker wound-healing process than the blank groups. The good wound-healing ability of the PDA-PAM hydrogel was attributed to four reasons. First, the high tissue adhesiveness of the
PDA-PAM hydrogel guaranteed intimate contact of the hydrogel to the surrounding tissue and protected the wound sites from infection during the entire healing period. Second, the excellent cell affinity of the hydrogel facilitated cell adhesion, attachment and migration. Third, the hydrogel had good affinity to extracellular matrix proteins (Supplementary Figure S17), and protein adsorption could further 
improve cell attachment, ${ }^{35}$ that established a positive feedback loop to finally create suitable extracellular matrix microenvironments for cell adhesion. ${ }^{36}$ Finally, the hydrogel stably immobilized EGF and preserved the bioactivity of EGF (Supplementary Figure S18) that enhanced the migration of fibroblasts to the wound area and promoted complete skin regeneration for the superior treatment of dermal wounds. The excellent wound-healing performance of the hydrogel shows great potential for clinic applications.

\section{Multifunctional hydrogels}

In addition to the high stretchability, stimuli-free self-healing ability and adhesiveness, the PDA-PAM hydrogel could serve as an excellent platform for tuning with multifunctionalities by hosting various nanobuilding blocks, because the PDA component in the hydrogel can facilitate the uniform distribution of functional NPs in the hydrogel by PDA grafting on the surface of the NPs (Supplementary Figure S22). The grafted NPs then interacted with the PAM network, and thus multifunctional hydrogels were obtained (Figure 7a). As examples, magnetic and conductive hydrogels were formed from the inclusion and dispersion of magnetic $\mathrm{Fe}_{3} \mathrm{O}_{4}$ and carbon black NPs into the hydrogels, respectively. In both cases, after PDA grafting, the NPs were well distributed in the water (Figures $7 \mathrm{~b}$ and $\mathrm{c}, \mathrm{i}$ ), and the hydrogel incorporated with NPs displayed a homogeneous morphology at the microscopic level, as observed by SEM, suggesting that the NPs were well dispersed into the polymeric matrix and covered with PDA microfibrils (Supplementary Figures S20 and S21, similar to the structure presented in the pure PDA-PAM hydrogel (Figure 2c).

A multifunctional conductive, stretchable, self-healable hydrogel was obtained after the incorporation of CB NPs (Supplementary Tables S4 and S5). The hydrogel showed good conductivity $\left(\sim 0.4 \mathrm{~S} \mathrm{~cm}^{-1}\right)$, as measured by the two-probe method (Supplementary Figure S20). The integration of multiple functions was well demonstrated in a battery-powered circuit with a lightemitting diode (LED) (Figure 7b,ii). The hydrogel worked as a conductor to connect the circuit to brightly light the LED. Then, the hydrogel was stretched, and the LED emission gradually weakened during this process. The resistance of the hydrogel under tension was measured that increased with an increase in extension ratio (Supplementary Figure S20e). The hydrogel was stretched to break, and the LED turned off. The fractured two parts of the hydrogel were brought into contact to self-heal for $2 \mathrm{~h}$, and the LED was brightly lit again. The healed hydrogel could once again bear large stretching.

A multifunctional magnetic, stretchable, adhesive hydrogel was obtained after the incorporation of $\mathrm{Fe}_{3} \mathrm{O}_{4}$ NPs. The magnetization curves showed that the hydrogel was a typical superparamagnetic material (Supplementary Figure S21c). To prove the integration of these hydrogel properties, hydrogel blocks with (black) and without (brown) $\mathrm{Fe}_{3} \mathrm{O}_{4}$ NPs were brought into contact and self-healed to form a hydrogel rope (Figure 7c,ii). Then, the hydrogel rope was adhered to nails and hung near a magnet. It was observed that the magnetic hydrogel regions (black part) were attracted by the magnet, and the hydrogel rope bent into a ' $\mathrm{S}$ ' shape.

\section{CONCLUSIONS}

The hydrogel was obtained by introducing reversible bonds homogeneously distributed inside a single covalent polymer (PAM) network. The PDA chains in the PAM network achieved a good balance between non-covalent bonds and covalent bonds in the hydrogel, the main reason for the toughness and self-healing properties. These results demonstrated that even a single network could achieve a tough hydrogel similar to previously reported double network and interpenetration hydrogels. This approach of designing hydrogels based on the hybrid crosslinking of a single polymer network with non-covalent bonds, such as hydrogen bonds, $\pi-\pi$ stacking, hydrophobic interactions and so on, could be generalized to produce a wide range of tough hydrogels with multiple characteristics.

In summary, we developed a novel but general design strategy to produce tough hydrogels, simultaneously showing the features of self-healing and tissue adhesiveness. The super stretchability and high fracture energy of this hydrogel were comparable with those of soft tissues. The self-healing ability rendered it particularly suitable for repairing fatigue-damaged tissues. The high cell affinity and tissue adhesiveness guaranteed it to fix to the surrounding tissues, thus promoting tissue regeneration. In addition, the hydrogel could serve as a universal platform to incorporate many types of functional NPs, resulting in hydrogels with multiple functionalities, such as magnetic and conductive response properties. The successful fabrication of conductive and magnetic hydrogels suggested that the PDA-PAM platform can be further extended to host other guest NPs with various therapeutic effects, such as drug- and growth factor-encapsulated polymeric or bioceramic NPs, greatly extending the range of applications of current hydrogels.

\section{CONFLICT OF INTEREST}

The authors declare no conflict of interest.

\section{ACKNOWLEDGEMENTS}

This work was financially supported by the National Key Research and Development Program of China (2016YFB0700802), the 863 Program (2015AA034202), the NSFC (81671824), the Open Fund of Key Lab of Advanced Technologies of Materials (MOE), the Fundamental Research Funds for the Central Universities (2682016CX075), the Start-up Funding of the South University of Science and Technology of China (F Ren), the 2015 Doctoral Innovation Funds of Southwest Jiaotong University, and Sichuan Province Youth Science and Technology Innovation Team (2016TD0026).

1 Seliktar, D. Designing cell-compatible hydrogels for biomedical applications. Science 336, 1124-1128 (2012)

2 Censi, R., Di Martino, P., Vermonden, T. \& Hennink, W. E. Hydrogels for protein delivery in tissue engineering. J. Controlled Release 161, 680-692 (2012).

3 Lee, K. Y. \& Mooney, D. J. Hydrogels for tissue engineering. Chem. Rev. 101, 1869-1880 (2001).

4 Hoffman, A. S. Hydrogels for biomedical applications. Adv. Drug Delivery Rev. 54, 3-12 (2002).

5 Calvert, P. Hydrogels for soft machines. Adv. Mater. 21, 743-756 (2009).

6 Zhao, X. Multi-scale multi-mechanism design of tough hydrogels: building dissipation into stretchy networks. Soft Matter 10, 672-687 (2014).

7 Gong, J. P. Why are double network hydrogels so tough? Soft Matter 6 , 2583-2590 (2010).

8 Haque, M. A., Kurokawa, T. \& Gong, J. P. Super tough double network hydrogels and their application as biomaterials. Polymer 53, 1805-1822 (2012).

9 Haraguchi, K. Synthesis and properties of soft nanocomposite materials with novel organic/inorganic network structures. Polym. J. 43, 223-241 (2011).

$10 \mathrm{Gong}$, J. P. Materials science. Materials both tough and soft. Science 344 161-162 (2014)

11 Sun, J. Y., Zhao, X., Illeperuma, W. R., Chaudhuri, O., Oh, K. H., Mooney, D. J., Vlassak, J. J. \& Suo, Z. Highly stretchable and tough hydrogels. Nature 489, 133-136 (2012).

12 Sun, T. L., Kurokawa, T., Kuroda, S., Ihsan, A. B., Akasaki, T., Sato, K., Haque, M. A. Nakajima, T. \& Gong, J. P. Physical hydrogels composed of polyampholytes demonstrate high toughness and viscoelasticity. Nat. Mater. 12, 932-937 (2013).

13 Yang, Y. \& Urban, M. W. Self-healing polymeric materials. Chem. Soc. Rev. 42, 7446-7467 (2013).

14 Zhao, Y., Nakajima, T., Yang, J. J., Kurokawa, T., Liu, J., Lu, J., Mizumoto, S., Sugahara, K., Kitamura, N., Yasuda, K., Daniels, A. U. \& Gong, J. P. Proteoglycans and glycosaminoglycans improve toughness of biocompatible double network hydrogels. Adv. Mater. 26, 436-442 (2014)

15 Chen, Y. M., Ogawa, R., Kakugo, A., Osada, Y. \& Gong, J. P. Dynamic cell behavior on synthetic hydrogels with different charge densities. Soft. Matter 5, 1804 (2009). 
16 Brandl, F., Sommer, F. \& Goepferich, A. Rational design of hydrogels for tissue engineering: impact of physical factors on cell behavior. Biomaterials 28, 134-146 (2007).

17 Drury, J. L. \& Mooney, D. J. Hydrogels for tissue engineering: scaffold design variables and applications. Biomaterials 24, 4337-4351 (2003).

18 Krogsgaard, M., Behrens, M. A., Pedersen, J. S. \& Birkedal, H. Self-healing musselinspired multi-pH-responsive hydrogels. Biomacromolecules 14, 297-301 (2013).

19 Kim, B. J., Oh, D. X., Kim, S., Seo, J. H., Hwang, D. S., Masic, A., Han, D. K. \& Cha, H. J. Mussel-mimetic protein-based adhesive hydrogel. Biomacromolecules 15, 1579-1585 (2014).

20 Brubaker, C. E., Kissler, H., Wang, L. J., Kaufman, D. B. \& Messersmith, P. B. Biological performance of mussel-inspired adhesive in extrahepatic islet transplantation. Biomaterials 31, 420-427 (2010).

21 Lee, H., Dellatore, S. M., Miller, W. M. \& Messersmith, P. B. Mussel-inspired surface chemistry for multifunctional coatings. Science 318, 426-430 (2007).

22 Lee, H., Lee, B. P. \& Messersmith, P. B. A reversible wet/dry adhesive inspired by mussels and geckos. Nature 448, 338-341 (2007).

23 Ahn, B. K., Lee, D. W., Israelachvili, J. N. \& Waite, J. H. Surface-initiated self-healing of polymers in aqueous media. Nat. Mater. 13, 867-872 (2014).

24 Holten-Andersen, N., Harrington, M. J., Birkedal, H., Lee, B. P., Messersmith, P. B. Lee, K. Y. \& Waite, J. H. Ph-induced metal-ligand cross-links inspired by mussel yield self-healing polymer networks with near-covalent elastic moduli. Proc. Natl. Acad. Sci. USA 108, 2651-2655 (2011).

25 Wilker, J. J. Self-healing polymers: sticky when wet. Nat. Mater. 13, 849-850 (2014).

26 Wei, Q., Zhang, F., Li, J., Li, B. \& Zhao, C. Oxidant-induced dopamine polymerization for multifunctional coatings. Polym. Chem. 1, 1430 (2010).

27 Ducrot, E., Chen, Y., Bulters, M., Sijbesma, R. P. \& Creton, C. Toughening elastomers with sacrificial bonds and watching them break. Science 344, 186-189 (2014).

$28 \mathrm{Liu}$, Y., Ai, K. \& Lu, L. Polydopamine and its derivative materials: synthesis and promising applications in energy, environmental, and biomedical fields. Chem. Rev. 114, 5057-5115 (2014)

29 Faure, E., Falentin-Daudré, C., Jérôme, C., Lyskawa, J., Fournier, D., Woisel, P. \& Detrembleur, C. Catechols as versatile platforms in polymer chemistry. Prog. Polym. Sci. 38, 236-270 (2013).

30 Xu, L. Q., Chen, J. C., Wang, R., Neoh, K.-G., Kang, E.-T. \& Fu, G. D. A poly (vinylidene fluoride)-graft-poly (dopamine acrylamide) copolymer for surface functionalizable membranes. RSC Adv. 3, 25204-25214 (2013).
31 He, S., Zhou, P., Wang, L., Xiong, X., Zhang, Y., Deng, Y. \& Wei, S. Antibiotic-decorated titanium with enhanced antibacterial activity through adhesive polydopamine for dental/bone implant. J. R. Soc. Interface 11, 20140169 (2014).

32 Kakuta, T., Takashima, Y., Nakahata, M., Otsubo, M., Yamaguchi, H. \& Harada, A. Preorganized hydrogel: self-healing properties of supramolecular hydrogels formed by polymerization of host-guest-monomers that contain cyclodextrins and hydrophobic guest groups. Adv. Mater. 25, 2849-2853 (2013).

33 Wang, X., Shi, J., Jiang, Z., Li, Z., Zhang, W., Song, X., Ai, Q. \& Wu, H. Preparation of ultrathin, robust protein microcapsules through template-mediated interfacial reaction between amine and catechol groups. Biomacromolecules 14, 3861-3869 (2013).

34 Czech, Z. Crosslinking of pressure sensitive adhesive based on water-borne acrylate. Polym. Int. 52, 347-357 (2003).

35 Lee, Y. B., Shin, Y. M., Lee, J.-H., Jun, I., Kang, J. K., Park, J.-C. \& Shin, H. Polydopamine-mediated immobilization of multiple bioactive molecules for the development of functional vascular graft materials. Biomaterials 33, 8343-8352 (2012).

36 Wang, Z., Wang, K., Zhang, Y., Jiang, Y., Lu, X., Fang, L., Gan, D., Lv, C., Zhang, H. \& $\mathrm{Qu}, \mathrm{S}$. Protein-affinitive polydopamine nanoparticles as an efficient surface modification strategy for versatile porous scaffolds enhancing tissue regeneration. Part. Part. Syst. Char. 33, 89-100 (2015).

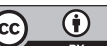

This work is licensed under a Creative Commons Attribution 4.0 International License. The images or other third party material in this article are included in the article's Creative Commons license, unless indicated otherwise in the credit line; if the material is not included under the Creative Commons license, users will need to obtain permission from the license holder to reproduce the material. To view a copy of this license, visit http:// creativecommons.org/licenses/by/4.0/

(C) The Author(s) 2017

Supplementary Information accompanies the paper on the NPG Asia Materials website (http://www.nature.com/am) 